\title{
Surgical outcomes of scaphoid fracture osteosynthesis with magnesium screws
}

\author{
Oktay Polat, $\mathrm{MD}^{1}\left(\mathbb{D}\right.$, Serdar Toy, $\mathrm{MD}^{1}$ (D), Birkan Kibar, $\mathrm{MD}^{2}(\mathbb{D}$ \\ 1Department of Orthopedics and Traumatology, Ağrı Training and Research Hospital, Ağrı, Turkey \\ 'Department of Orthopedics and Traumatology, Haydarpaşa Numune Training and Research Hospital, Istanbul, Turkey
}

The scaphoid is vital for the proper mechanics of wrist function. Scaphoid fractures account for two-thirds of all carpal fractures. ${ }^{[1,2]}$ The most common mechanism of injury is a fall of an outstretched arm/hand with an axial impact on a pronated, ulnar-deviated, and hyper-dorsiflexed wrist. ${ }^{[3]}$

A single headless compression screw fixation along the central longitudinal axis of the scaphoid is the most commonly used surgical technique for scaphoid fractures. ${ }^{[4]}$ Screw removal rates of 3 to $14 \%$ have been reported after scaphoid surgeries in the literature. ${ }^{[5-7]}$ Implant removal after fixation with titanium implants is a challenging clinical problem in orthopedic surgery. Therefore, absorbable implants have been evaluated for years. Absorbable implants have been introduced in orthopedic surgery to prevent potential complications related to implant removal. Most of the bioabsorbable implants used currently are polymer-based implants. ${ }^{[8]}$ However,

Received: June 09, 2021

Accepted: August 31, 2021

Published online: November 19, 2021

Correspondence: Oktay Polat, MD. Ağrı Eğitim ve Araştırma Hastanesi, Ortopedi ve Travmatoloji Kliniği, 04200 Ağrı, Türkiye.

E-mail: pltkrdsı@@hotmail.com

Doi: $10.52312 / j d r s .2021 .298$

Citation: Polat O, Toy S, Kibar B. Surgical outcomes of scaphoid fracture osteosynthesis with magnesium screws. Jt Dis Relat Surg 2021;32(3):721-728

(02021 All right reserved by the Turkish Joint Diseases Foundation

This is an open access article under the terms of the Creative Commons Attribution-NonCommercial License, which permits use, distribution and reproduction in any medium, provided the original work is properly cited and is not used for commercial purposes (http://creativecommons.org/licenses/by-nc/4.0/).

\section{ABSTRACT}

Objectives: This study aims to evaluate the mid-term functional and radiological outcomes of magnesium-based screws in the treatment of scaphoid fractures.

Patients and methods: Between February 2015 and February 2018, a total of 21 patients (18 males, 3 females; mean age: $28.5 \pm 5.8$ years; range, 19 to 39 years) with acute scaphoid waist fractures who underwent fracture fixation with biologically degradable magnesium-based compression screws were retrospectively analyzed. Fractures were classified according to the Herbert and Fisher classification. The absence of pain on palpation and painless active range of motion were accepted as the signs of union.

Results: The mean follow-up was $43.3 \pm 5.3$ (range, 36 to 52 ) months. According to the Herbert and Fisher classification, nine patients had type B1 and 12 patients had type B2 scaphoid fractures. Union was achieved in all cases. The mean time to union was $11.2 \pm 1.5$ (range, 9 to 14 ) weeks. The mean grip strength, flexion, and extension were $43.57^{\circ}, 73.57^{\circ}$, and $76.43^{\circ}$, respectively. The grip strength, pinch strength, and range of motion of the operated side were evaluated at the final follow-up visit and compared with the contralateral side (control group). No complication occurred. Any screw was not removed.

Conclusion: Magnesium-based compression screws can be safely used for acute scaphoid fractures considering their favorable functional and radiological results.

Keywords: Absorbable implants, fracture fixation, internal fixation, magnesium, scaphoid bone.

many undesirable reactions and complications, such as osteolysis, foreign body reaction, and cyst formation, have been described using these implants. ${ }^{[8,9]}$ Due to these reasons, magnesium $(\mathrm{Mg})$-based implants, which are involved in bone structure and bone metabolism, have been developed. The degradation products of these implants are not toxic. Other advantages of these implants include high stability, osteoconductive effect, infection-inhibiting effect, and fewer artifacts in computed tomography (CT) or magnetic resonance imaging. ${ }^{[10-13]}$ 
Several studies have reported reliable clinical results regarding the $\mathrm{Mg}$ screws used in many orthopedic surgeries, ${ }^{[13-16]}$ A study on scaphoid fracture osteosynthesis with $\mathrm{Mg}$ screws reported a few cases and short-term clinical outcomes. In the present study, we aimed to investigate whether $\mathrm{Mg}$ screws could be used effectively and safely in treating scaphoid fractures and to evaluate the mid-term functional and radiological outcomes of $\mathrm{Mg}$ screws in treating scaphoid fractures.

\section{PATIENTS AND METHODS}

This single center, retrospective review was conducted at Haydarpaşa Numune Training and Research Hospital, Department of Orthopedics and Traumatology between February 2015 and February 2018. A total of 25 patients with acute scaphoid fractures who underwent fracture fixation with bioabsorbable $\mathrm{Mg}$ headless compression screws (3.2 mm) (MAGNEZIX ${ }^{\circledast}$ CS, Syntellix AG, Hanover, Germany) and were followed for at least 36 months were included. Patients whose scaphoid fracture fixation was augmented with other implants such as titanium screws or Kirschner wires (K-wires) were excluded from the study. One patient who was unable to be contacted and three patients who were lost to follow-up were also excluded. Finally, a total of 21 patients (18 males, 3 females; mean age: $28.5 \pm 5.8$ years; range, 19 to 39 years) were included. Fractures were classified according to the Herbert and Fisher classification. ${ }^{[17]} \mathrm{A}$ written informed consent was obtained from each patient. The study protocol was approved by the Ağrı Training and Research Hospital Institutional Review Board (IRB Approval No. 2021-210/17.07). The study was conducted in accordance with the principles of the Declaration of Helsinki.

The volar approach was used for all fractures. The technical aspects of the procedure were described earlier by Inoue and Tamura ${ }^{[18]}$ and Ledoux et al. ${ }^{[19]}$ Surgery was performed under general or regional anesthesia. Fractures were reduced with closed reduction using an image intensifier. A Kirschner wire (K-wire) guide was introduced percutaneously. Then, bioabsorbable Mg headless compression screw fixation was performed and confirmed using an image intensifier. In all cases, a scaphoid cast was applied postoperatively with a window at the surgical wound site for wound dressing. Two weeks after the operation, the sutures and casts were removed. Under the supervision of a physiotherapist, the patients were, then, encouraged to exercise with a wrist immobilizer brace for four weeks. The absence of pain on palpation and painless active range of motion were accepted as the signs of union.

Handgrip strengthening exercise with activeassisted range of motion exercises of the wrist was started. After eight weeks, strengthening exercises were initiated in patients who did not have pain on palpation. Another physiotherapist in the study center performed strengthening exercises.

The institutional patient database was used to access all radiological data in the Picture Archiving and Communication System (PACS), patients' records, medical history, surgical documents, and follow-up visit notes. Demographic data, diagnostic tests, and radiological results were collected. The following variables were recorded: age, dominance, preoperative delay, return of occupational and sports activities, union time, follow-up term, union delay, and complications. Grip strength, pinch strength, and range of motion of the operated wrist were evaluated at the final follow-up visit and compared with the contralateral side (control group). Anteroposterior and lateral radiographs of the wrist were taken in the neutral, extended, and flexed positions.

\section{Statistical analysis}

Statistical analysis was performed using the IBM SPSS for Windows version 23.0 software (IBM Corp., Armonk, NY, USA). Descriptive data were expressed in mean \pm standard deviation (SD), median (min-max), or number and frequency, where applicable. The Shapiro-Wilk test was used to determine whether continuous data were distributed normally between groups. The results of the Shapiro-Wilk test showed that data did not have normal distributions. The Wilcoxon test was used to compare the groups, and Spearman correlation test was used to analyze the relationship of functional scores. Functional results were evaluated with the intraclass correlation coefficient (ICC) test. A $p$ value of $<0.05$ was considered statistically significant.

\section{RESULTS}

According to the Herbert and Fisher classification, nine $(42.9 \%)$ patients had type B1 and $12(57.1 \%)$ patients had type B2 scaphoid fractures. Union was achieved in all cases (100\%). The mean time to union was $11.2 \pm 1.5$ (range, 9 to 14) weeks (Table I).

The mean follow-up was $43.3 \pm 5.3$ (range, 36 to 52 ) months. Differences between the operated and nonoperated wrists were significant in grip strength, 


\begin{tabular}{|c|c|c|c|c|c|}
\hline \multicolumn{6}{|c|}{$\begin{array}{c}\text { TABLE I } \\
\text { Demographic data of patients }\end{array}$} \\
\hline & $\mathrm{n}$ & $\%$ & Mean \pm SD & Median & Min-Max \\
\hline Age (year) & & & $28.5 \pm 5.8$ & 26 & $19-39$ \\
\hline \multicolumn{6}{|l|}{ Sex } \\
\hline Male & 18 & 85.7 & & & \\
\hline Female & 3 & 14.3 & & & \\
\hline \multicolumn{6}{|l|}{ Side } \\
\hline Right & 14 & 66.7 & & & \\
\hline Left & 7 & 33.3 & & & \\
\hline \multicolumn{6}{|l|}{ Smoking } \\
\hline Yes & 10 & 47.6 & & & \\
\hline No & 11 & 52.4 & & & \\
\hline \multicolumn{6}{|l|}{ Dominance } \\
\hline Right & 19 & 90.5 & & & \\
\hline Left & 2 & 9.5 & & & \\
\hline \multicolumn{6}{|l|}{ Mechanism of trauma } \\
\hline Simple falls & 17 & 81.0 & & & \\
\hline Traffic accident & 3 & 14.3 & & & \\
\hline Sports injuries & 1 & 4.8 & & & \\
\hline \multicolumn{6}{|l|}{ Herbert and fisher classification } \\
\hline B1 & 9 & 42.9 & & & \\
\hline B2 & 12 & 57.1 & & & \\
\hline Mean time to union (week) & & & $11.2 \pm 1.5$ & 11 & $9-14$ \\
\hline Follow-up (month) & & & $43.3 \pm 5.3$ & 44 & $36-52$ \\
\hline Mean preoperative delay (day) & & & $3.0 \pm 2.3$ & 2 & $1-8$ \\
\hline
\end{tabular}

\begin{tabular}{|c|c|c|c|c|c|c|c|}
\hline & & omparison & $\begin{array}{c}\text { TABL } \\
\text { e operate }\end{array}$ & contralat & ide & & \\
\hline & Ope & side & Contr & al side & Wilcoxon & Spearr & 's rho \\
\hline & Median & Min-Max & Median & Min-Max & $p$ & r spearman & $p$ \\
\hline Grip strength & 43 & $35-60$ & 44 & $37-58$ & $<0.001^{* *}$ & 0.962 & $<0.001^{* *}$ \\
\hline Flexion & 75 & $60-80$ & 75 & $70-80$ & $0.008^{*}$ & 0.872 & $<0.001^{* *}$ \\
\hline Extension & 80 & $65-80$ & 80 & $70-80$ & 0.317 & 0.762 & $<0.001^{* *}$ \\
\hline Ulnar deviation & 25 & $20-30$ & 30 & $25-35$ & $<0.001^{* *}$ & 0.590 & $0.005^{*}$ \\
\hline Radial deviation & 15 & $10-20$ & 20 & $20-25$ & $<0.001^{\star *}$ & 0.142 & 0.540 \\
\hline Pinch & 7 & $5-9$ & 8 & $6-9$ & $<0.001^{\star *}$ & 0.882 & $<0.001^{* *}$ \\
\hline
\end{tabular}

\begin{tabular}{|c|c|c|c|c|c|}
\hline \multicolumn{6}{|c|}{$\begin{array}{c}\text { TABLE III } \\
\text { Intraclass correlation coefficient }\end{array}$} \\
\hline \multirow[b]{2}{*}{ Variables } & & \multirow[b]{2}{*}{$\begin{array}{l}\text { Intraclass } \\
\text { correlation }\end{array}$} & \multicolumn{2}{|c|}{ 95\% Confidence interval } & \multirow[b]{2}{*}{$p$} \\
\hline & & & $\begin{array}{l}\text { Lower } \\
\text { bound }\end{array}$ & $\begin{array}{l}\text { Upper } \\
\text { bound }\end{array}$ & \\
\hline Grip strength (operated side) & Grip strength (contralateral side) & 0.978 & 0.945 & 0.991 & $<0.001^{* *}$ \\
\hline Flexion (operated side) & Flexion (contralateral side) & 0.748 & 0.379 & 0.989 & $0.002^{*}$ \\
\hline Extension (operated side) & Extension (contralateral side) & 0.862 & 0.661 & 0.944 & $<0.001^{* *}$ \\
\hline Ulnar deviation (operated side) & Ulnar deviation (contralateral side) & 0.717 & 0.301 & 0.885 & $0.003^{*}$ \\
\hline Radial deviation (operated side) & Radial deviation (contralateral side) & 0.292 & -0.746 & 0.713 & 0.224 \\
\hline Pinch (operated side) & Pinch (contralateral side) & 0.938 & 0.847 & 0.975 & $<0.001^{* *}$ \\
\hline
\end{tabular}



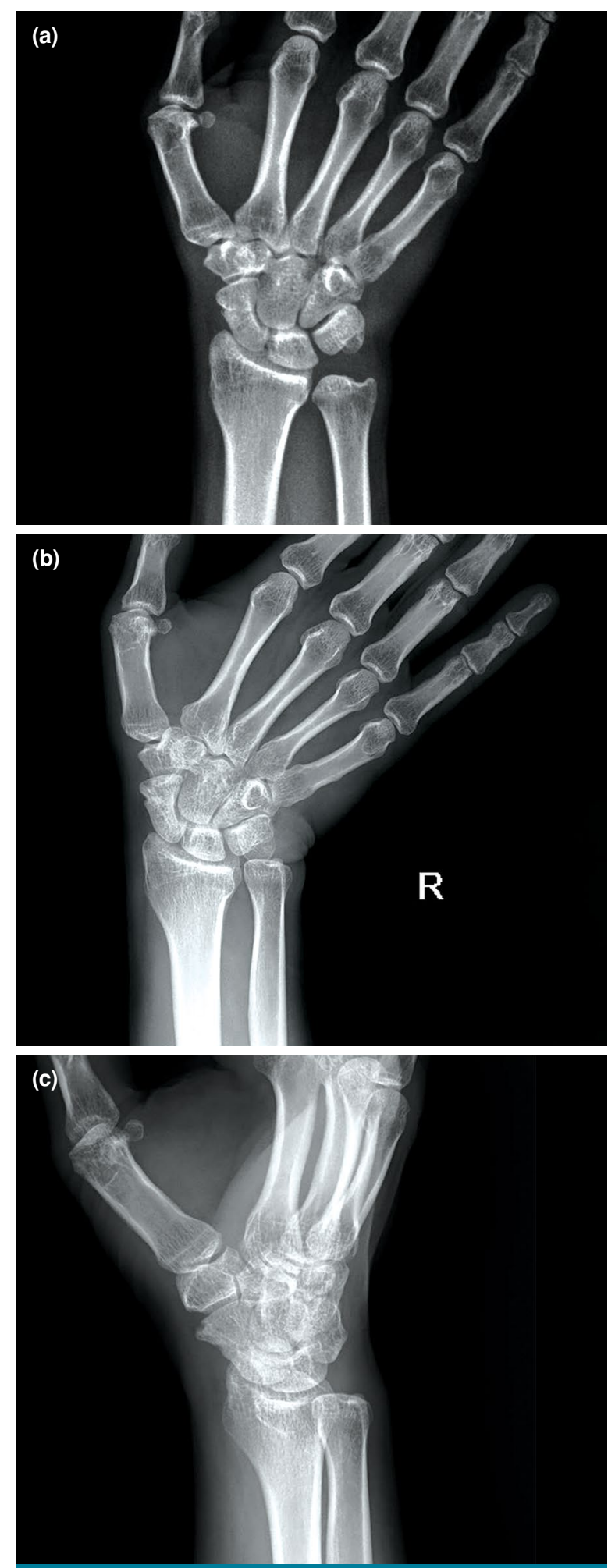

FIGURE 1. (a) A 33-year-old male patient had a scaphoid fracture-preoperative anteroposterior (AP) radiograph. (b) At the postoperative four-year follow-up, there was no gas shadow on the AP X-ray, and a faded screw silhouette was present. (c) At the postoperative four-year follow-up, no gas shadow was detected on the oblique $\mathrm{X}$-ray, and a faded screw silhouette was current. flexion, ulnar deviation, radial deviation, and pinch $(p<0.05)$. However, no significant difference was found between the extension of the wrist on the operated side and the normal side $(\mathrm{p}=0.317)$ (Table II).

A strong correlation was found between the operated and contralateral sides in terms of grip strength $(r=0.962, p<0.001)$, flexion $(r=0.872, p<0.001)$, extension $(r=0.762, p<0.001)$, ulnar deviation $(r=0.590$, $\mathrm{p}=0.005)$, and pinch strength $(\mathrm{r}=0.882, \mathrm{p}<0.001)$ (Table II). A strong correlation was noted between the operated and contralateral sides in terms of grip strength $(\mathrm{r}=0.978, \mathrm{p}<0.001)$, flexion $(\mathrm{r}=0.748, \mathrm{p}=0.002)$, extension $(\mathrm{r}=0.862, \mathrm{p}<0.001)$, ulnar deviation $(\mathrm{r}=0.717$, $\mathrm{p}=0.003)$, and pinch strength $(\mathrm{r}=0.938, \mathrm{p}<0.001)$ (Table III). No complication was encountered. The screw was not removed in any case.

Given the retrospective nature of this study, scheduled radiographs of all patients were not obtained at similar dates. All available serial follow-up radiographs and CT scans (if available) were evaluated to monitor the degradation of bioabsorbable $\mathrm{Mg}$ screws. In the early postoperative radiographs, gas shadowing occurred in the soft tissue and bone around the implant in all patients (Figure 1). These shadows started to fade at nine months of follow-up, and barely any was left at the end of 18 months (Figure 2). In the late period, these gas silhouettes completely disappeared in the trabecular structure of the bone in CT scans and radiographs (Figure 1, 2).

\section{DISCUSSION}

In the present study, we evaluated the radiological and functional outcomes of patients who underwent osteosynthesis for acute scaphoid waist fractures with bioabsorbable $\mathrm{Mg}$ screws using the volar percutaneous technique. No malunion, nonunion, or any other complications developed in any of the patients. Favorable functional results were obtained compared to the opposite hand. Owing to the absorbable properties of bioabsorbable $\mathrm{Mg}$ screws used in this study, screw irritation and removal did not occur. With these screws, no patient needed a second surgical intervention.

To the best of our knowledge, very few clinical studies have utilized $\mathrm{Mg}$ screws so far. May et al. ${ }^{[13]}$ performed surgery with $\mathrm{Mg}$ screws in 23 patients for medial malleolus fractures. In their study, fracture union was observed in all patients, and second surgery for implant removal was not needed. Moreover, Mg screws were like titanium screws in terms of functional and radiological outcomes, and 

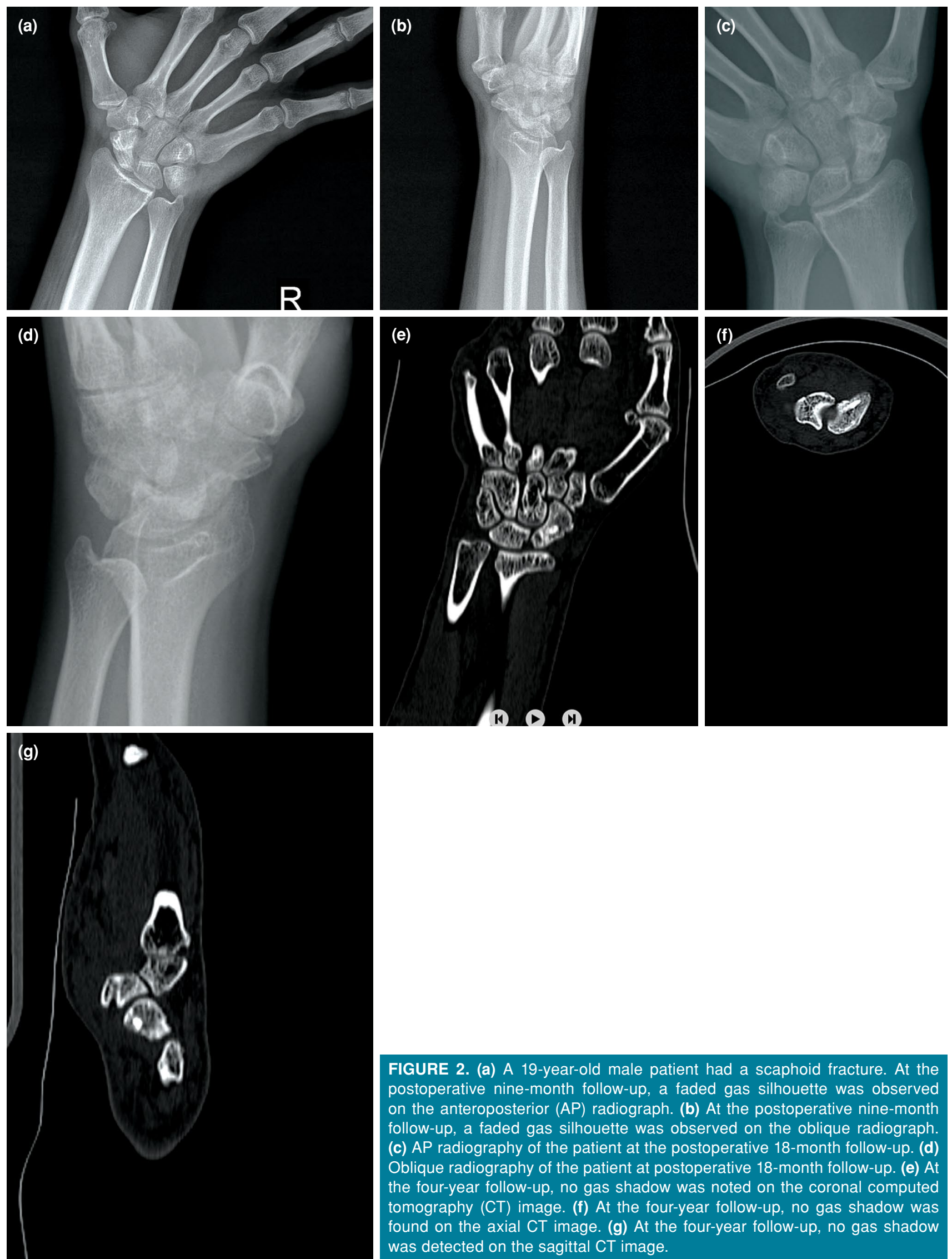

FIGURE 2. (a) A 19-year-old male patient had a scaphoid fracture. At the postoperative nine-month follow-up, a faded gas silhouette was observed on the anteroposterior (AP) radiograph. (b) At the postoperative nine-month follow-up, a faded gas silhouette was observed on the oblique radiograph. (c) AP radiography of the patient at the postoperative 18-month follow-up. (d) Oblique radiography of the patient at postoperative 18-month follow-up. (e) At the four-year follow-up, no gas shadow was noted on the coronal computed tomography (CT) image. (f) At the four-year follow-up, no gas shadow was found on the axial CT image. (g) At the four-year follow-up, no gas shadow was detected on the sagittal CT image. 
their complication rates were lower than titanium screws. Windhagen et al. ${ }^{[14]}$ evaluated 13 patients who underwent chevron osteotomy for hallux valgus surgery with $\mathrm{Mg}$ screws and reported that union was achieved in all patients. In addition, no foreign body reaction, osteolysis, or systemic inflammatory reaction was observed in any of their patients. Biber et al. ${ }^{[15]}$ performed surgery with an $\mathrm{Mg}$ screw on a patient with a lateral malleolus fracture. They noted that the union was achieved at three months, radiolucent areas formed around the screw in the first months, and these areas disappeared at 17 months of follow-up. Grieve et al. ${ }^{[20]}$ performed surgery with $\mathrm{Mg}$ screws in six patients for scaphoid fractures $(n=3)$ and carpal fractures $(n=3)$. They reported that recovery was achieved at 12 weeks, and there were no complications. Baldini et al. ${ }^{[21]}$ reported that clinical and radiological outcomes were good, and no side effects were observed in 14 children treated with an $\mathrm{Mg}$ screw. In our study, union was achieved in all patients, and no complications developed in any patient. Therefore, in the present study, bioabsorbable $\mathrm{Mg}$ screws could provide adequate fixation for acute scaphoid waist fractures. Surgeries performed with $\mathrm{Mg}$ screws do not require second surgery for implant removal.

Compression screws placed along the scaphoid central longitudinal axis are preferred for internal fixation of scaphoid waist fractures. ${ }^{[4]}$ Different screw options are available for internal fixation, such as Herbert screw, 3.0- $\mathrm{mm}$ headless compression screws, and Acutrak ${ }^{\circledR}$ (Acumed Inc., OR, USA) screws. ${ }^{[22]}$ After these screws were used for scaphoid fractures, implant removal surgery was performed between 4 and 14\%. ${ }^{[5-7,23]}$ Implant removal surgeries pose a significant burden on the healthcare economy and carry the risks of infection, cartilage damage, scarring, and potential neurovascular injury. ${ }^{[24-26]}$ Ibrahim et al. ${ }^{[27]}$ published a meta-analysis of patients who underwent surgery for acute scaphoid fractures. In their study of 182 patients, Acutrack ${ }^{\circledR}$ screws were used in 66 and Herbert screws were used in 116 patients, and union was achieved in 179 patients. They reported some complications, such as screw irritation, infection, complex regional pain syndrome, and scarring. Moreover, implant removal surgeries were needed due to screw irritation. To the best of our knowledge, surgeons may encounter difficulties in acute scaphoid fractures, such as nonunion, implant removal due to screw irritation, and infection. In our study, we did not encounter these problems due to the osteoinductive, infection-inhibiting effects, and resorption of $\mathrm{Mg}$ screws.

Many adverse reactions and complications, such as osteolysis, foreign body reaction, and cyst formation, have been reported with polymer-based implants. ${ }^{[28]}$ These reactions and complications occur, as these polymer-based implants are first hydrolyzed and separated into monomers by an enzymatic reaction. These monomers, then, enter the citric acid cycle and are excreted as water and carbon dioxide from the body. During enzymatic hydrolysis, the local $\mathrm{pH}$ decreases, and an acidic environment is formed around the implants. ${ }^{[28]}$ The $\mathrm{Mg}$ screws participate in the electrochemical reaction and turn into $\mathrm{Mg}(\mathrm{OH})_{2}$ and hydrogen $\left(\mathrm{H}_{2}\right)$ which can be seen as radiolucency in soft and bone tissues. Subsequently, these radiolucent areas disappear. ${ }^{[11,13,29]}$ Adverse events with the use of bioabsorbable $\mathrm{Mg}$ screws were reported in the literature. Meier and Panzica ${ }^{[30]}$ performed surgery with Mg-based compression screws in five patients with acute scaphoid waist fractures. These patients were followed for one year. In these patients, union was achieved, and physical activity was allowed at six months of follow-up. The wrist scores of their patients were excellent (95 to 100\%) after one year of follow-up, and no clinical complications were reported. However, three of five patients had resorption cysts in radiological examinations at three months of follow-up. These resorption cysts were seen on radiographs, until the sixth month of follow-up. These findings are the normal degradation process of $\mathrm{Mg}$ screws, as previously mentioned. Leigheb et al. ${ }^{[31]}$ conducted a systematic review on the efficacy and safety of the Mg-based osteosynthesis materials and reported these materials had similar efficacy and safety with titanium screws and that the most common effect was gas formation. Our study findings are consistent with the literature: radiolucent areas were found in the initial follow-up, and these areas disappeared after the union was achieved. The clinical and radiological outcomes of the patients were excellent at the final follow-up visit.

Meier and Panzica ${ }^{[30]}$ reported that patients with scaphoid fractures treated with an $\mathrm{Mg}$ screw had excellent (95 to 100\%) wrist scores after one year of follow-up. Rettig et al. ${ }^{[32]}$ surgically treated their patients with scaphoid fractures with a Herbert screw. All their patients had functional wrist range of motion (wrist extension, 57 ; wrist flexion, $52^{\circ}$ ) and grip strength in $85 \%$ of the contralateral side. The functional results in our study were compatible with the literature: grip strength, 95\%; flexion, 
96\%; extension, 99\%; ulnar deviation, $88 \%$; radial deviation, 77\%; and pinch, $92 \%$ compared to the opposite side.

Nonetheless, this study has some limitations. First, it has a retrospective design. Second, there is no control group, and the sample size is relatively small. Third, only Herbert type B1 and B2 scaphoid fractures were included. Finally, the mean time to union was evaluated from the available radiographs. However, the main strength of this study is that the mean follow-up period was $43.3 \pm 5.3$ months, which allowed us to assess mid-term outcomes, including post-traumatic osteoarthritis.

In conclusion, $\mathrm{Mg}$-based compression screws can be safely used for acute scaphoid fractures. Redo surgery is not needed for implant removal, as the Mg-based compression screws dissolve over time. Further large-scale, long-term, prospective clinical studies are needed to confirm these findings.

\section{Declaration of conflicting interests}

The authors declared no conflicts of interest with respect to the authorship and/or publication of this article.

\section{Funding}

The authors received no financial support for the research and/or authorship of this article.

\section{REFERENCES}

1. Acar B, Köse Ö, Turan A, Katı YA, Güler F. Single versus double screw fixation for the treatment of scaphoid waist fractures: Finite element analysis and preliminary clinical results in scaphoid nonunion. Jt Dis Relat Surg 2020;31:73-80.

2. Kılıç B, Çalışkan M, Agar A, Uzun B, Ertem F, Gülabi D, et al. Comparison of two different screw trajectories in the treatment of oblique scaphoid fractures: A mechanical study on composite bone models. Jt Dis Relat Surg 2021;32:377-82.

3. Fowler JR, Hughes TB. Scaphoid fractures. Clin Sports Med 2015;34:37-50.

4. Clementson M, Jørgsholm P, Besjakov J, Thomsen N, Björkman A. Conservative treatment versus arthroscopicassisted screw fixation of scaphoid waist fractures--a randomized trial with minimum 4-year follow-up. J Hand Surg Am 2015;40:1341-8.

5. Gregory JJ, Mohil RS, Ng AB, Warner JG, Hodgson SP. Comparison of Herbert and Acutrak screws in the treatment of scaphoid non-union and delayed union. Acta Orthop Belg 2008;74:761-5.

6. Radford PJ, Matthewson MH, Meggitt BF. The Herbert screw for delayed and non-union of scaphoid fractures: A review of fifty cases. J Hand Surg Br 1990;15:455-9.

7. Dias JJ, Brealey SD, Fairhurst C, Amirfeyz R, Bhowal $\mathrm{B}$, Blewitt $\mathrm{N}$, et al. Surgery versus cast immobilisation for adults with a bicortical fracture of the scaphoid waist (SWIFFT): A pragmatic, multicentre, open-label, randomised superiority trial. Lancet 2020;396:390-401.
8. Raikin SM, Ching AC. Bioabsorbable fixation in foot and ankle. Foot Ankle Clin 2005;10:667-84.

9. Böstman OM, Pihlajamäki HK. Adverse tissue reactions to bioabsorbable fixation devices. Clin Orthop Relat Res 2000;(371):216-27.

10. Seitz, JM, Lucas A, Kirschner M. Magnesium-based compression screws: A novelty in the clinical use of implants. JOM 2016;68:1177-82.

11. Waizy H, Diekmann J, Weizbauer A, Reifenrath J, Bartsch I, Neubert V, et al. In vivo study of a biodegradable orthopedic screw (MgYREZr-alloy) in a rabbit model for up to 12 months. J Biomater Appl 2014;28:667-75.

12. Rahim MI, Eifler R, Rais B, Mueller PP. Alkalization is responsible for antibacterial effects of corroding magnesium. J Biomed Mater Res A 2015;103:3526-32.

13. May H, Alper Kati Y, Gumussuyu G, Yunus Emre T, Unal M, Kose O. Bioabsorbable magnesium screw versus conventional titanium screw fixation for medial malleolar fractures. J Orthop Traumatol 2020;21:9.

14. Windhagen H, Radtke K, Weizbauer A, Diekmann J, Noll Y, Kreimeyer U, et al. Biodegradable magnesium-based screw clinically equivalent to titanium screw in hallux valgus surgery: Short term results of the first prospective, randomized, controlled clinical pilot study. Biomed Eng Online 2013;12:62.

15. Biber R, Pauser J, Brem M, Bail HJ. Bioabsorbable metal screws in traumatology: A promising innovation. Trauma Case Rep 2017;8:11-5.

16. Plaass C, von Falck C, Ettinger S, Sonnow L, Calderone F, Weizbauer A, et al. Bioabsorbable magnesium versus standard titanium compression screws for fixation of distal metatarsal osteotomies - 3 year results of a randomized clinical trial. J Orthop Sci 2018;23:321-7.

17. Herbert TJ, Fisher WE. Management of the fractured scaphoid using a new bone screw. J Bone Joint Surg [Br] 1984;66:114-23.

18. Inoue G. Closed technique for the Herbert screw insertion in an undisplaced fracture of the scaphoid. J Orthop Surg Tech 1991;6:1-7.

19. Ledoux P, Chahidi N, Moermans JP, Kinnen L. Percutaneous Herbert screw osteosynthesis of the scaphoid bone. Acta Orthop Belg 1995;61:43-7.

20. Grieve P, O'Carroll S, Albastaki O. Six cas de série de patients de Magnezix®. Une vis métallique absorbable pour la fixation de la fracture du carpe et des fusions entre les carpes. Hand surgery and rehabilitation 2017;36:488-9.

21. Baldini M, Coppa V, Falcioni D, Senigagliesi E, Marinelli M, Gigante AP. Use of resorbable magnesium screws in children: Systematic review of the literature and short-term follow-up from our series. J Child Orthop 2021;15:194-203.

22. Fowler JR, Ilyas AM. Headless compression screw fixation of scaphoid fractures. Hand Clin 2010;26:351-61.

23. Pelto-Vasenius K, Hirvensalo E, Böstman O, Rokkanen P. Fixation of scaphoid delayed union and non-union with absorbable polyglycolide pin or Herbert screw. Consolidation and functional results. Arch Orthop Trauma Surg 1995;114:347-51.

24. Hanson B, van der Werken C, Stengel D. Surgeons' beliefs and perceptions about removal of orthopaedic implants. BMC Musculoskelet Disord 2008;9:73. 
25. Sie TH, Abdel-Kader KF, Allcock S. A useful technique for removal of Herbert screws from the scaphoid. J Hand Surg Br 1998;23:332-3.

26. Reith G, Schmitz-Greven V, Hensel KO, Schneider MM, Tinschmann T, Bouillon B, et al. Metal implant removal: benefits and drawbacks--a patient survey. BMC Surg 2015;15:96.

27. Ibrahim T, Qureshi A, Sutton AJ, Dias JJ. Surgical versus nonsurgical treatment of acute minimally displaced and undisplaced scaphoid waist fractures: Pairwise and network meta-analyses of randomized controlled trials. J Hand Surg Am 2011;36:1759-68.

28. Kontakis GM, Pagkalos JE, Tosounidis TI, Melissas J, Katonis P. Bioabsorbable materials in orthopaedics. Acta Orthop Belg 2007;73:159-69.
29. Kamrani S, Fleck C. Biodegradable magnesium alloys as temporary orthopaedic implants: a review. Biometals 2019;32:185-93.

30. Meier R, Panzica M. First results with a resorbable MgYREZr compression screw in unstable scaphoid fractures show extensive bone cysts. Handchir Mikrochir Plast Chir 2017;49:37-41.

31. Leigheb M, Veneziano M, Tortia R, Bosetti M, Cochis A, Rimondini $\mathrm{L}$, et al. Osteosynthesis devices in absorbable Magnesium alloy in comparison to standard ones: A systematic review on effectiveness and safety. Acta Biomed 2021;92:e2021025.

32. Rettig ME, Kozin SH, Cooney WP. Open reduction and internal fixation of acute displaced scaphoid waist fractures. J Hand Surg Am 2001;26:271-6. 